\title{
Impact of Organic, Inorganic and Biofertilizers on Crop Yield and N, P and K Uptake under Rainfed Maize-Wheat Cropping System
}

\author{
Bhoye Ranjanabai Chhagan, M.P. Sharma, K.R. Sharma, Abhijit Samanta, \\ Owais Ali Wani*, Dileep Kachroo, Manish Kumar, V.K. Razdan, Vikas Sharma, \\ A.K. Mondal and V.M. Arya \\ Division of Soil Science and Agricultural Chemistry, Sher-e-Kashmir University of \\ Agricultural Sciences and Technology, Chatha, Jammu- 180009 (J\&K), India \\ *Corresponding author
}

\section{Keywords \\ Maize-wheat cropping system, Integrated nutrient management, Crop yield and Nutrient uptake}

Article Info

Accepted:

17 March 2019

Available Online:

10 April 2019

\section{A B S T R A C T}

Integrated effect of organic, inorganic and biofertilizers on crop yield and N,P and $\mathrm{K}$ uptake under rainfed maize-wheat cropping system and available nutrients during 20152016 is being studied in sandy loam at Jammu under the INM maize-wheat trial. The results revealed that the application of recommended levels of NPK to maize-wheat with FYM, VC and biofertilizers (Azotobacter and phosphate solubilizing bacteria) resulted in grain 66.53 per cent and straw 13.00 per cent increase over control in maize and wheat yields, respectively. Increasing levels of $50 \% \mathrm{~N}+$ recom. $\mathrm{P}+\mathrm{K}+\mathrm{S}+\mathrm{Zn}+\mathrm{B}$ + Vermicompost + Azotobactera + Phosphorus Solubilizing Bacteria significantly increased the yield of both the crops. Integrated use of organic, inorganic and biofertilizers improved the soil status of available N, P, K, S, $\left(116.09,14.22,124.61,9.41 \mathrm{~kg} \mathrm{ha}^{-1}\right), \mathrm{Zn}$ and B $(0.56$ and $0.48 \mathrm{mg} \mathrm{kg}^{-1}$ ), over the initial values. A declining trend (N 70.05, P 9.14, K110.13, S $8.14 \mathrm{~kg} \mathrm{ha}^{-1} \mathrm{Zn} 0.36 \mathrm{mg} \mathrm{kg}^{-1}$ and $0.34 \mathrm{~kg} \mathrm{ha}^{-1}$ ) from the initial value of available nutrients. Increasing level of total uptake in maize $\mathrm{N}, \mathrm{P}, \mathrm{K}, \mathrm{S}, \mathrm{Zn}$ and $\mathrm{B}$. This indicates a considerable mining of INM from the soil and suggest the need to adopt judicious organic, inorganic and biofertilizers.

\section{Introduction}

Rain fed agriculture contributes $58 \%$ of global food basket and constitutes $66 \%$ of the net sown area in India. Site specific nutrient management has received considerable attention due to potential benefits of increasing input use efficiency, improving economic margins of crop production and reducing environmental risks. Hence, a comprehensive understanding of spatial variability of soil properties is becoming increasingly essential in agriculture as soil properties vary from field to a large region scale and are influenced by geology, topography climate as well. The Kandi belt consisting of sub mountainous area of Jammu region arising from Punjab plains with gentle 
slope of nearly three degrees and touching with low hilly Siwalik system of rocks lies in the outer Himalayas of Jammu region. The soils of this region are of lithosols type having undulated topography and scrub forest. Loss of organic matter, whether by erosion or high temperature in the rainfed agro-ecosystem, adds, to improvement of soil resources of several elements essential for plants growth. A decline in organic matter multiplies nutrient deficiency, it falls by the two-thirds symbolizes a serious suppression in nutrient availability. In addition, fertilizer consumption in rainfed areas is very low. The challenge of improving productivity in rainfed areas can be addressed by efficient utilization of available nutrients. Efficient nutrient management demands understanding the pathways of nutrient losses through gaseous loss, leaching loss, erosion and runoff losses and developing technologies to minimize these losses. Many water-soluble nutrients are lost through run off during intense rainfall and nutrients absorbed on the surface of soil particles-clays and silts and soil organic matter are lost when the top soil is eroded by water or wind. These losses of nutrients are not merely economic losses but may cause serious environmental problems and hence must be controlled by developing appropriate site-specific technologies. The native available nutrients should be optimally allocated among the crops to get maximum returns by allowing optimization of nutrient production functions which relate the crop responses to applied nutrients under given soil, climate, and management factors under rainfed conditions. To avoid any risk, the fertilizer recommendation in the rainfed region should be made only in the linear response range. Fertilizer allocation to crops based on soil test and crop correlation under rainfed condition for achieving targeted yield can help in improving nutrient use efficiency by crops. The yield targets can be decided based on availability of water other inputs and financial condition of the farmer depending on the inherent particular nutrient status of the soils (Sharma et al., 2017).

\section{Materials and Methods}

The present investigation is a part of an ongoing experiment with maize-wheat cropping system in progress since kharif and rabi 2015 and 2016 at Advance center for rainfed Agriculture Rakh Dhainsar, SKUAST-Jammu and Department of Soil Science and Agricultural Chemistry, Chatha, Jammu Jammu and Kashmir ( $32^{\circ} 39^{\prime} \mathrm{N}$ and $\left.74^{0} 58 \mathrm{E}\right)$. Dhainsar, Jammu has a sub-tropical climate with a characteristic feature dry and cold winter. In winter season i.e. Oct to March the temperature 2 to $200 \mathrm{c}$ and the relative humidity 41 to $65 \%$.Gernerally, dry and warm weather prevails during the months of March to June. The temperature in the month of May reaches as high as 48 0C. Monsoon season extends from first of July to mid-September. Total annual rainfall varies from $1049.2 \mathrm{~mm}$ to $1304 \mathrm{~mm}$ with the mean value of around $\mathrm{mm}$. The length of growing period of both the crops ranges from 200 to 210 days. The soil of the experimental field is sandy loam in texture having $\mathrm{pH}$ (6.67), Bulk density(1.64 Mg m-3), Water holding capacity $(11.01 \%)$, Cation exchange capacity (8.14 C mol(p+) $\left(\mathrm{kg}^{-1}\right)$, organic carbon $2.05 \mathrm{~g}$ $\mathrm{kg}^{-1}$ and available nitrogen $(\mathrm{N})\left(70 \mathrm{~kg} \mathrm{ha}^{-1}\right)$, Phosphorus (P) (9.14), Potassium (K) 110.13 $\mathrm{kg} \mathrm{ha}^{-1}$ were, Sulphur (S) (8.14 $\left.\mathrm{kg} \mathrm{ha}^{-1}\right)$ and Zinc (Zn) (0.36 $\left.\mathrm{mg} \mathrm{kg}^{-1}\right)$, Boron (B) $(0.34 \mathrm{mg}$ $\left.\mathrm{kg}^{-1}\right)$, respectively. There were eleven treatments viz., T1, Control, T2. Recommended NPK, T3 Reco. NPK+ S + Zn $+\mathrm{B}$, T4 50\% $\mathrm{N}+$ Reco. $\mathrm{P}+\mathrm{K}+\mathrm{S}+\mathrm{Zn}+\mathrm{B}+$ $50 \% \mathrm{~N}$ through FYM, T5 50\% N + Reco. P + $\mathrm{K}+\mathrm{S}+\mathrm{Zn}+\mathrm{B}+50 \% \mathrm{~N}$ through VC, T6 $75 \% \mathrm{~N}+$ Reco. $\mathrm{P}+\mathrm{K}+\mathrm{S}+\mathrm{Zn}+\mathrm{B}+25 \% \mathrm{~N}$ through FYM, T7 75\% $\mathrm{N}+$ Reco. $\mathrm{P}+\mathrm{K}+\mathrm{S}$ $+\mathrm{Zn}+\mathrm{B}+25 \% \mathrm{~N}$ through VC, T8 $50 \% \mathrm{~N}+$ Reco. $\mathrm{P}+\mathrm{K}+\mathrm{S}+\mathrm{Zn}+\mathrm{B}+50 \% \mathrm{~N}$ through 
$\mathrm{FYM}+$ Azot +PSB, T9 50\% N + Reco. P +K $+\mathrm{S}+\mathrm{Zn}+\mathrm{B}+50 \% \mathrm{~N}$ through $\mathrm{VC}+\mathrm{Azot}+$ $\mathrm{PSB}, \mathrm{T} 1075 \% \mathrm{~N}+\mathrm{Reco} . \mathrm{P}+\mathrm{K}+\mathrm{S}+\mathrm{Zn}+\mathrm{B}+25 \%$ $\mathrm{N}$ through FYM +Azto +PSB, T11 75\% N + Reco. $\mathrm{P}+\mathrm{K}+\mathrm{S}, \mathrm{Zn}+\mathrm{B}+25 \% \mathrm{~N}$ through $\mathrm{VC}$ + Azot + PSB. Each treatment was replicated thrice in a randomized block design. The recommended $\mathrm{P}, \mathrm{K}, \mathrm{S}, \mathrm{Zn}$ and $\mathrm{B}$ doses, based on initial soil test, were $70.05 \mathrm{~kg}$ ha-1, 9.14 $\mathrm{kg} \mathrm{ha}^{-1}, 110.13 \mathrm{~kg} \mathrm{ha}^{-1}, 8.14 \mathrm{~kg} \mathrm{ha}^{-1}, 0.36 \mathrm{mg}$ $\mathrm{kg}^{-1}$ and $0.34 \mathrm{mg} \mathrm{kg}^{-1}$, $\mathrm{kg} \mathrm{ha}^{-1}$,maize and wheat. The sources of $\mathrm{N}$ through FYM and VC were incorporated treatment. (Sowing time) in the soil sowing time of maize and wheat during kharif and rabi season since 2015-16. Treatments T8, T9, T10 and T11 involved inoculation by Azotobacter and PSB culture (@5g kg-1 seed). Maize- wheat varieties used were Vivak Maize-25 and PBW-175 respectively, maize in general was sown on the set of monsoon (third week of July) as rainfed crop during kharif and wheat in the to fourth week of October as irrigation crop during rabi. The maturity and yield data were recorded after harvested at maturity and yield data were recorded after threshing. Soil samples from $0-15 \mathrm{~cm}$ depth were collected after harvest of maize in the 2 th cropping year (2015-16 and 2017) and were analyzed for available $\mathrm{N}$ by alkaline permanganate method (Subbiah and Asija, 1956), available P (Olsen et al., 1954) and available $\mathrm{K}$ (ammonium acetate extract).All observations were recorded for both the crops and soil properties were analyzed statistically.

1. Grain yield $\left(\mathrm{kg} \mathrm{ha}^{-1}\right)=$

Yield obtained from net plot $(\mathrm{kg}) \times 10,000$

Area of net plot

2. Nutrient uptake $\left(\mathrm{kg} \mathrm{ha}^{-1}\right)=$

Nutrient content $(\%)$ x dry matter accumulation $\left(\mathrm{kg} \mathrm{ha}^{-1}\right)$

100

\section{Results and Discussion}

The highest grain and straw yield of maize during 2015-16 was recorded in $\mathrm{T}_{9}(28.51)$ to lowest in $\mathrm{T}_{1}(17.12)$ and $\mathrm{T}_{9}$ (87.90) and lowest in $\mathrm{T}_{1}$ (77.79). The higher yield under $\mathrm{T}_{9}$ might be due to faster mineralization of $\mathrm{VC}$ and beneficial effects of Azotobacter and PSB application for seed treatment might be due to balanced addition of NPK, N, P, K, S, Zn, B under $\mathrm{T}_{2}(24.23)$ and $\mathrm{T}_{3}(25.63)$ and integrated effect of organic, inorganic and biofertilizer which enhanced nutrients availability and resulted improvement grain yield in $\mathrm{T}_{5}(26.54)$ and $\mathrm{T}_{8}(27.87)$. These observations are in line with those reported by Khandre et al., 2015. Beneficial effects of Azotobacter and PSB application in wheat and have been reported by Singh and Prasad 2011, Madhu et al., 2012 also obtained similar observation. However low yield in $\mathrm{T}_{1}$ could be due to scanty availability of nutrients which resulted lead to lower yield in both years

The grain and straw yield of wheat found significantly highest in $\mathrm{T}_{9}(19.90)$ to lowest in $\mathrm{T}_{1}$ (8.03) and $\mathrm{T}_{9}(37.95)$ to lowest in $\mathrm{T}_{1}$ (18.62). It might be due to speedy mineralization of vermicompost and potential role of azotobacter and PSB. Whereas lowest grain yield in T1(8.03) could be due to less availability of nutrients. Khandare et al., 2015 reported similar increases in grain yield due to Azotobacter and PSB application in combination with organic and inorganic and might be due to balanced addition of NPK and integrated uses of organic, inorganic and biofertilizers which influenced the availability of nutrients resulted in continuous improvement in grain yield of wheat. These observations are similar to those reported by Thakur et al., (2011).

The highest uptake of $\mathrm{N}$ in grain and straw uptake of maize was found in $\mathrm{T}_{9}$ (49.95) to lowest in $\mathrm{T}_{1}$ (22.05) and straw of maize was 
observed in $\mathrm{T}_{9}$ (51.20) and lowest in $\mathrm{T}_{1}$ (23.23) it might be due to balanced addition of NPK, N, P, K, S, Zn, B and combined uses of organics, inorganics and biofertilizers which directly add the nutrient and solubilized native form of nutrient. These observations were supported by Thangasamy et al., (2017) and Mishra et al., (2008) while studying $\mathrm{N}$ uptake in maize-wheat cropping system. However, $\mathrm{N}$ uptake under treatment $\mathrm{T}_{3}, \mathrm{~T}_{4}, \mathrm{~T}_{5}, \mathrm{~T}_{7}, \mathrm{~T}_{8}$ and $\mathrm{T}_{11}$ were observed at par to each other. It may be due to poor mineralization of $\mathrm{N}$ in soil resulted low uptake. These findings supported by Thangasamy et al., (2017). The highest total uptake of $\mathrm{N}$ in grain plus straw of maize was recorded in $\mathrm{T}_{9}(101.15)$ to lowest in $\mathrm{T}_{1}(45.28)$. The highest to uptake of total $\mathrm{N}$ in grain plus straw might be due to combined addition of organic (VC) inorganic and biofertilizer which contributes nitrogen as well as solubilized native form of nutrient from soil. However, lowest uptake of $\mathrm{N}$ in grain plus straw may be due to scanty amount of $\mathrm{N}$ in the treatment (Chaterjee et al., 2017).

The highest $\mathrm{N}$ uptake in grain yield of wheat was obtained in $T_{9}(17.82)$ to lowest in $\mathrm{T}_{1}(8.99)$ and The highest total $\mathrm{N}$ uptake in grain plus straw of wheat was found in $\mathrm{T}_{9}(51.02)$ and lowest in $\mathrm{T}_{1}(21.39)$. The highest total $\mathrm{N}$ uptake in grain plus straw might be due to integrated addition of organic, inorganic and biofertilizers which enhanced $\mathrm{N}$ availability in soil other by increased $\mathrm{N}$ uptake. Similar finding was also reported by Biswas et al., (2017).

The highest $\mathrm{P}$ uptake in grain yield of maize was observed in $T_{9}$ (12.93) to lowest in $\mathrm{T}_{1}(5.19)$ and The highest $\mathrm{P}$ uptake in straw yield of maize has been observed in $\mathrm{T}_{9}(17.30)$ to lowest in $\mathrm{T}_{1}(12.89)$. The total $\mathrm{P}$ uptake in grain plus straw yield of maize had been observed highest in $\mathrm{T}_{9}(30.23)$ to lowest in T1(18.08). The highest $P$ uptake in grain plus straw in $\mathrm{T}_{9}$ due to combined addition of organic, inorganic and biofertilizer including VC and PSB which directly add the same nutrient as well as influenced its availability by solubilizing native form in soils thereby enhanced P uptake. These results support the observation of Thangasamy et al., (2017).

The highest $\mathrm{P}$ uptake in grain yield of wheat was observed in $\mathrm{T}_{9}(17.82)$ to lowest in $\mathrm{T}_{1}(8.99)$ and The highest $\mathrm{P}$ uptake in straw yield of wheat was noticed in $\mathrm{T}_{9}(33.20)$ to lowest in $T_{1}(12.40)$ The highest $P$ uptake in grain plus straw in wheat was recorded in $\mathrm{T}_{9}(51.02)$ to lowest in $\mathrm{T}_{1}(21.39)$. The highest $P$ uptake in grain plus straw in $T_{9}(51.02)$ might be due to combined addition of organic, inorganic and biofertilizers including Azotobacter, PSB which directly adds and influenced its availability thereby resulted increased uptake. Whereas, lowest P uptake in $\mathrm{T}_{1}(21.39)$ can be due to its low availability, thereby decreased its uptake. These observations are in agreement with those reported by Thangasamy et al., (2017), Chesti et al., (2013).

The highest $\mathrm{P}$ uptake in grain yield of maize was observed in $T_{9}$ (12.93) to lowest in $\mathrm{T}_{1}(5.19)$ The highest $\mathrm{P}$ uptake in straw yield of maize has been observed in $\mathrm{T}_{9}(17.30)$ and lowest in $\mathrm{T}_{1}(12.89)$, The total $\mathrm{P}$ uptake in grain plus straw yield of maize had been observed highest in $\mathrm{T}_{9}(30.23)$ and lowest in T1(18.08), The highest $\mathrm{P}$ uptake in grain plus straw in $\mathrm{T}_{9}$ due to combined addition of organic, inorganic and biofertilizer including VC and PSB which directly add the same nutrient as well as influenced its availability by solubilizing native form in soils thereby enhanced $\mathrm{P}$ uptake. These results support the observation of Thangasamy et al., (2017).

The highest $\mathrm{P}$ uptake in grain yield of wheat was observed in $\mathrm{T}_{9}(17.82)$ to lowest in $\mathrm{T}_{1}(8.99)$ and The highest $\mathrm{P}$ uptake in straw 
yield of wheat was noticed in $T_{9}(33.20)$ to lowest in $\mathrm{T}_{1}(12.40)$ The highest $\mathrm{P}$ uptake in grain plus straw in wheat was recorded in $\mathrm{T}_{9}(51.02)$ to lowest in $\mathrm{T}_{1}(21.39)$.

The highest $\mathrm{P}$ uptake in grain plus straw in $\mathrm{T}_{9}(51.02)$ might be due to combined addition of organic, inorganic and biofertilizers including Azotobacter, PSB which directly adds and influenced its availability thereby resulted increased uptake. Whereas, lowest $P$ uptake in $\mathrm{T}_{1}(21.39)$ can be due to its low availability, thereby decreased its uptake. These observations are in agreement with those reported by Thangasamy et al., 2017,
Chesti et al., 2013. The highest total K uptake in grain plus straw of maize was noticed in $\mathrm{T}_{9}$ (128.80) to lowest in $\mathrm{T}_{1}(92.36)$ and the highest $\mathrm{K}$ uptake in grain yield of wheat was observed in $\mathrm{T}_{9}(55.02)$ and lowest in $\mathrm{T}_{1}(20.85)$.

The total $\mathrm{K}$ uptake in grain plus straw was highest in $\mathrm{T}_{9}(177.62)$ and lowest in $\mathrm{T}_{1}(74.83)$ The highest $\mathrm{K}$ uptake in grain yield of maize and wheat may be due to integrated addition of organic, inorganic and biofertilizers which influenced its availability in soil (Fig. 1-5 and Table

$1-8)$.

Table.1 Integrated effect of inorganic, organic and biofertilizers on grain and straw yield (q ha $\left.{ }^{-1}\right)$ after harvesting of maize crop

\begin{tabular}{|c|c|c|c|c|c|c|c|}
\hline \multirow[t]{2}{*}{ Sr.No } & \multirow{2}{*}{ Treatments } & $\begin{array}{c}I^{\text {st }} \\
\text { year }\end{array}$ & $\begin{array}{l}\text { II }{ }^{\text {nd }} \\
\text { year }\end{array}$ & $\begin{array}{l}\text { Overa } \\
\text { ll }\end{array}$ & $\begin{array}{l}I^{\text {st }} \\
\text { year }\end{array}$ & $\begin{array}{l}\text { II }{ }^{\text {nd }} \\
\text { year }\end{array}$ & Overall \\
\hline & & \multicolumn{3}{|c|}{$\left(\mathrm{q} \mathrm{ha}^{1}\right)^{2}$} & \multicolumn{3}{|c|}{$\left(\mathrm{q} \mathrm{ha}^{-1}\right)$} \\
\hline T1 & Control & 17.12 & 18.25 & 17.69 & 77.79 & 80.86 & 79.33 \\
\hline T2 & Recommended NPK & 24.23 & 25.13 & 24.68 & 79.12 & 83.29 & 81.21 \\
\hline T3 & Reco. $\mathrm{NPK}+\mathrm{S}+\mathrm{Zn}+\mathrm{B}$ & 25.63 & 26.34 & 25.99 & 84.74 & 86.38 & 85.56 \\
\hline T4 & $\begin{array}{l}50 \% N+\text { Reco. } P+K+S+Z n+B \\
+50 \% N \text { through FYM }\end{array}$ & 25.50 & 26.74 & 26.12 & 84.90 & 86.25 & 85.58 \\
\hline T5 & $\begin{array}{l}50 \% N+\operatorname{Reco.~} P+K+S+Z n+B \\
+50 \% N \text { through } V C\end{array}$ & 26.54 & 27.46 & 27.00 & 85.62 & 88.49 & 87.06 \\
\hline T6 & $\begin{array}{l}\text { 75\% } \mathbf{N}+\operatorname{Reco.} \mathbf{P}+\mathbf{K}+\mathrm{S}+\mathrm{Zn}+ \\
\mathbf{B}+25 \% \text { N through FYM }\end{array}$ & 25.50 & 26.74 & 26.12 & 83.58 & 84.38 & 83.98 \\
\hline T7 & $\begin{array}{l}75 \% \mathbf{N}+\operatorname{Reco.} \mathbf{P}+\mathrm{K}+\mathrm{S}+\mathrm{Zn} \\
+\mathrm{B}+25 \% \mathrm{~N} \text { through VC }\end{array}$ & 25.99 & 27.29 & 26.64 & 83.44 & 85.24 & 84.34 \\
\hline T8 & $\begin{array}{l}\mathbf{5 0 \%} \mathbf{N}+\text { Reco. } \mathrm{P}+\mathrm{K}+\mathrm{S}+\mathrm{Zn}+\mathbf{B}+ \\
\mathbf{5 0 \%} \mathrm{N} \text { through FYM }+ \text { Azot }+\mathrm{PSB}\end{array}$ & 27.87 & 28.66 & 28.27 & 86.33 & 89.03 & 87.68 \\
\hline T9 & $\begin{array}{l}\mathbf{5 0 \%} \mathbf{N}+\operatorname{Reco} . \mathrm{P}+\mathrm{K}+\mathrm{S}+\mathrm{Zn}+\mathrm{B}+ \\
\mathbf{5 0 \%} \mathrm{N} \text { through } \mathrm{VC}+\mathbf{A z o t}+\mathrm{PSB}\end{array}$ & 28.51 & 29.35 & 28.93 & 87.90 & 89.86 & 88.88 \\
\hline T10 & $\begin{array}{l}\mathbf{7 5 \%} \mathrm{N}+\text { Reco.P }+\mathrm{K}+\mathrm{S}+\mathrm{Zn}+\mathrm{B}+ \\
\mathbf{2 5 \%} \mathrm{N} \text { through } \mathrm{FYM}+\mathrm{Azto}+\mathrm{PSB}\end{array}$ & 27.56 & 28.53 & 28.05 & 85.21 & 85.60 & 86.41 \\
\hline T11 & $\begin{array}{l}\text { 75\% } \mathbf{N}+\operatorname{Reco.} \mathbf{P}+\mathrm{K}+\mathrm{S}, \mathrm{Zn}+\mathrm{B}+ \\
25 \% \mathrm{~N} \text { through } \mathrm{VC}+\mathrm{Azot}+\mathrm{PSB}\end{array}$ & 27.16 & 27.47 & 27.32 & 85.95 & 86.92 & 86.44 \\
\hline & \pm S.E.(m) & 0.23 & 0.23 & 0.14 & 0.35 & 0.39 & 0.57 \\
\hline & C.D. $(P=0.05)$ & 0.67 & 0.70 & 0.45 & 1.04 & 1.18 & 1.81 \\
\hline
\end{tabular}


Table.2 Integrated effect of inorganic, organic and biofertilizers on grain and straw yield (q ha $\left.{ }^{-1}\right)$ after harvesting of wheat crop

\begin{tabular}{|c|c|c|c|c|c|c|c|}
\hline \multirow[t]{2}{*}{ Sr.No } & \multirow[t]{2}{*}{ Treatments } & $\begin{array}{c}\mathbf{I}^{\text {st }} \\
\text { year }\end{array}$ & $\begin{array}{l}\text { II }^{\text {nd }} \\
\text { year }\end{array}$ & $\begin{array}{l}\text { Overa } \\
\text { ll }\end{array}$ & $\begin{array}{c}\mathbf{I}^{\text {st }} \\
\text { year }\end{array}$ & $\begin{array}{l}\text { II }^{\text {nd }} \\
\text { year }\end{array}$ & $\begin{array}{l}\text { Over } \\
\text { all }\end{array}$ \\
\hline & & \multicolumn{3}{|c|}{$\left(q\right.$ ha- $\left.^{1}\right)$} & \multicolumn{3}{|c|}{$\left(q h^{-1}\right)$} \\
\hline $\mathbf{T 1}$ & Control & 7.31 & 8.03 & 7.67 & 18.62 & 20.29 & 19.46 \\
\hline $\mathbf{T} 2$ & Recommended NPK & 13.78 & 14.61 & 14.20 & 24.89 & 31.36 & 28.13 \\
\hline T3 & Reco. $\mathbf{N P K}+\mathbf{S}+\mathbf{Z n}+\mathbf{B}$ & 14.76 & 15.63 & 15.20 & 31.05 & 34.19 & 32.62 \\
\hline T4 & $\begin{array}{l}\mathbf{5 0 \%} \mathrm{N}+\text { Reco. } \mathrm{P}+\mathrm{K}+\mathrm{S}+ \\
\mathrm{Zn}+\mathrm{B}+\mathbf{5 0 \%} \mathrm{N} \text { through } \\
\text { FYM }\end{array}$ & 15.29 & 16.40 & 15.85 & 34.20 & 35.59 & 34.90 \\
\hline T5 & $\begin{array}{l}50 \% \mathrm{~N}+\text { Reco. } \mathrm{P}+\mathrm{K}+\mathrm{S}+ \\
\mathrm{Zn}+\mathrm{B}+\mathbf{5 0 \%} \mathrm{N} \text { through VC }\end{array}$ & 16.63 & 17.25 & 17.19 & 37.04 & 36.20 & 36.62 \\
\hline T6 & $\begin{array}{l}75 \% \mathrm{~N}+\operatorname{Reco} . \mathrm{P}+\mathrm{K}+\mathrm{S}+\mathrm{Zn} \\
+\mathrm{B}+\mathbf{2 5 \%} \mathrm{N} \text { through FYM }\end{array}$ & 14.47 & 15.68 & 15.08 & 37.07 & 34.89 & 35.98 \\
\hline T7 & $\begin{array}{l}75 \% \mathrm{~N}+\text { Reco. } \mathrm{P}+\mathrm{K}+\mathrm{S}+\mathrm{Zn} \\
+\mathrm{B}+25 \% \mathrm{~N} \text { through } \mathrm{VC}\end{array}$ & 14.87 & 15.96 & 15.42 & 37.95 & 37.01 & 37.48 \\
\hline T8 & $\begin{array}{l}50 \% \mathrm{~N}+\text { Reco. P+ K +S +Zn } \\
+\mathrm{B}+\mathbf{5 0 \%} \mathrm{N} \text { through FYM } \\
+ \text { Azot }+ \text { PSB }\end{array}$ & 17.45 & 18.50 & 17.98 & 37.27 & 38.75 & 38.01 \\
\hline T9 & $\begin{array}{l}50 \% \mathrm{~N}+\text { Reco. } \mathrm{P}+\mathrm{K}+\mathrm{S}+\mathrm{Zn} \\
+\mathrm{B}+50 \% \mathrm{~N} \text { through } \mathrm{VC}+ \\
\text { Azot }+ \text { PSB }\end{array}$ & 19.04 & 19.90 & 19.47 & 38.00 & 40.39 & 39.20 \\
\hline T10 & $\begin{array}{l}75 \% \mathrm{~N}+\text { Reco.P }+\mathrm{K}+\mathrm{S}+\mathrm{Zn}+ \\
\mathrm{B}+25 \% \mathrm{~N} \text { through } \mathrm{FYM} \\
+ \text { Azto }+\mathrm{PSB}\end{array}$ & 14.24 & 15.38 & 14.63 & 35.50 & 37.43 & 36.47 \\
\hline T11 & $\begin{array}{l}75 \% \mathrm{~N}+\text { Reco. } \mathrm{P}+\mathrm{K}+\mathrm{S}, \mathrm{Zn} \\
+\mathrm{B}+25 \% \mathrm{~N} \text { through VC }+ \\
\text { Azot }+ \text { PSB }\end{array}$ & 15.02 & 16.23 & 15.63 & 37.37 & 39.84 & 38.61 \\
\hline & \pm S.E.(m) & 0.34 & 0.32 & 0.09 & 1.90 & 0.38 & 1.24 \\
\hline & C.D. $(P=0.05)$ & 1.01 & 0.95 & 0.27 & 6.06 & 1.13 & 3.96 \\
\hline
\end{tabular}


Table.3 Integrated effect of inorganic, organic and biofertilizers on nitrogen uptake $\left(\mathrm{kg} \mathrm{ha}^{-1}\right)$ in grain, straw and total uptake after harvesting of maize crop

\begin{tabular}{|c|c|c|c|c|c|c|c|c|c|}
\hline \multirow[t]{2}{*}{ Sr.No } & \multirow[t]{2}{*}{ Treatments } & $\begin{array}{c}\text { Grain } \\
\left(I^{\text {st }}\right. \\
\text { year })\end{array}$ & \multicolumn{2}{|c|}{$\begin{array}{c}\text { Straw } \\
\left(\mathbf{I}^{\text {st }} \text { year }\right)\end{array}$} & $\begin{array}{l}\text { Total } \\
\text { upta } \\
\text { ke }\end{array}$ & $\begin{array}{c}\text { Grain } \\
\left(\text { II Id }^{\text {d }}\right. \\
\text { year })\end{array}$ & $\begin{array}{l}\text { Straw } \\
\text { (II } \\
\text { year) }\end{array}$ & \multicolumn{2}{|c|}{$\begin{array}{l}\text { Total } \\
\text { uptake }\end{array}$} \\
\hline & & \multicolumn{4}{|c|}{$\left(\operatorname{kg~ha}^{1}\right)$} & \multicolumn{4}{|c|}{$\left(\mathrm{kg} \mathrm{ha}^{-1}\right)$} \\
\hline T1 & Control & 22.05 & 23.23 & 45.28 & 24.39 & 23.72 & 48.11 & 22.05 & 23.23 \\
\hline T2 & Recommended NPK & 33.51 & 23.99 & 57.50 & 35.55 & 26.09 & 61.64 & 33.51 & 23.99 \\
\hline T3 & Reco. $\mathbf{N P K}+\mathrm{S}+\mathrm{Zn}+\mathbf{B}$ & 41.60 & 32.17 & 73.77 & 43.29 & 33.35 & 76.64 & 41.60 & 32.17 \\
\hline T4 & $\begin{array}{l}50 \% \mathrm{~N}+\operatorname{Reco.} \mathrm{P}+\mathrm{K}+\mathrm{S}+ \\
\mathrm{Zn}+\mathrm{B}+\mathbf{5 0 \%} \mathrm{N} \text { through } \\
\text { FYM }\end{array}$ & 45.78 & 39.67 & 85.45 & 48.63 & 40.99 & 89.62 & 45.78 & 39.67 \\
\hline T5 & $\begin{array}{l}\mathbf{5 0 \%} \mathbf{N}+\operatorname{Reco.} \mathbf{P}+\mathbf{K}+\mathbf{S}+ \\
\mathbf{Z n}+\mathbf{B}+\mathbf{5 0 \%} \mathbf{N} \text { through } \mathbf{V C}\end{array}$ & 47.95 & 45.85 & 93.80 & 48.87 & 48.31 & 97.18 & 47.95 & 45.85 \\
\hline T6 & $\begin{array}{l}75 \% \mathbf{N}+\text { Reco. } \mathbf{P}+\mathbf{K}+\mathbf{S}+\mathbf{Z n} \\
+\mathbf{B}+25 \% \text { N through FYM }\end{array}$ & 44.88 & 40.88 & 85.76 & 46.83 & 42.06 & 88.89 & 44.88 & 40.88 \\
\hline T7 & $\begin{array}{l}75 \% \mathbf{N}+\text { Reco. } \mathbf{P}+\mathrm{K}+\mathrm{S}+\mathrm{Zn} \\
+\mathrm{B}+25 \% \mathrm{~N} \text { through } \mathrm{VC}\end{array}$ & 46.57 & 44.03 & 90.60 & 47.56 & 51.19 & 98.75 & 46.57 & 44.03 \\
\hline T8 & $\begin{array}{l}50 \% \mathrm{~N}+\text { Reco. } \mathrm{P}+\mathrm{K}+\mathrm{S}+\mathrm{Zn}+ \\
\mathrm{B}+50 \% \mathrm{~N} \text { through FYM } \\
+ \text { Azot }+\mathrm{PSB}\end{array}$ & 45.89 & 47.23 & 93.12 & 47.51 & 51.82 & 99.33 & 45.89 & 47.23 \\
\hline T9 & $\begin{array}{l}50 \% \mathrm{~N}+\text { Reco. } \mathrm{P}+\mathrm{K}+\mathrm{S}+\mathrm{Zn} \\
+\mathrm{B}+50 \% \mathrm{~N} \text { through } \mathrm{VC}+ \\
\text { Azot }+ \text { PSB }\end{array}$ & 49.95 & 51.20 & $\begin{array}{l}101.1 \\
5\end{array}$ & 50.67 & 53.12 & $\begin{array}{l}103.7 \\
9\end{array}$ & 49.95 & 51.20 \\
\hline T10 & $\begin{array}{l}\text { 75\% N+Reco.P +K +S }+\mathrm{Zn}+ \\
\text { B+ 25\% N through FYM } \\
+ \text { Azto +PSB }\end{array}$ & 42.51 & 43.85 & 86.36 & 46.49 & 47.08 & 93.57 & 42.51 & 43.85 \\
\hline T11 & $\begin{array}{l}75 \% \mathrm{~N}+\operatorname{Reco.} \mathrm{P}+\mathrm{K}+\mathrm{S}, \mathrm{Zn}+ \\
\text { B+ 25\% N through VC + Azot } \\
+\mathrm{PSB}\end{array}$ & 47.25 & 47.16 & 94.41 & 47.21 & 49.83 & 97.04 & 47.25 & 47.16 \\
\hline & \pm S.E.(m) & 0.88 & 1.07 & 1.95 & 0.69 & 0.77 & 1.45 & 0.88 & 1.07 \\
\hline & C.D. $(\mathbf{P}=\mathbf{0 . 0 5})$ & 2.62 & 3.16 & 5.77 & 2.03 & 2.28 & 4.30 & 2.62 & 3.16 \\
\hline
\end{tabular}


Table.4 Integrated effect of inorganic, organic and biofertilizers on nitrogen uptake $\left(\mathrm{kg} \mathrm{ha}^{-1}\right)$ in grain, straw and total uptake after harvesting of wheat crop

\begin{tabular}{|c|c|c|c|c|c|c|c|c|c|}
\hline \multirow[t]{2}{*}{ Sr.No } & \multirow[t]{2}{*}{ Treatments } & \multicolumn{2}{|c|}{$\begin{array}{c}\text { Grain } \\
\left(\mathbf{I}^{\text {st }} \text { year }\right)\end{array}$} & $\begin{array}{c}\text { Straw } \\
\left(\mathbf{I}^{\text {st }} \text { year }\right)\end{array}$ & $\begin{array}{l}\text { Total } \\
\text { uptake }\end{array}$ & $\begin{array}{l}\text { Grain } \\
\left(\text { II }^{\text {nd }}\right. \\
\text { nan }\end{array}$ & $\begin{array}{l}\text { Stras } \\
\left(I^{n}{ }^{n}\right. \\
\text { vagr }\end{array}$ & \multicolumn{2}{|c|}{$\begin{array}{l}\text { Total } \\
\text { uptake }\end{array}$} \\
\hline & & \multicolumn{4}{|c|}{$\left(\right.$ kg ha- $\left.{ }^{1}\right)$} & \multicolumn{4}{|c|}{$\left(\mathrm{kg} \mathrm{ha}^{-1}\right)$} \\
\hline T1 & Control & 8.99 & 12.40 & 21.39 & 8.38 & 13.71 & 22.09 & 8.99 & 12.40 \\
\hline T2 & Recommended NPK & 14.42 & 23.74 & 38.16 & 13.97 & 25.13 & 39.1 & 14.42 & 23.74 \\
\hline T3 & Reco. $\mathrm{NPK}+\mathrm{S}+\mathrm{Zn}+\mathrm{B}$ & 15.84 & 25.82 & 41.66 & 15.57 & 27.19 & 42.76 & 15.84 & 25.82 \\
\hline T4 & $\begin{array}{l}\mathbf{5 0 \%} \mathbf{N}+\text { Reco. } \mathbf{P}+\mathrm{K}+\mathrm{S}+ \\
\mathrm{Zn}+\mathrm{B}+\mathbf{5 0 \%} \mathrm{N} \text { through } \\
\text { FYM }\end{array}$ & 17.16 & 27.05 & 44.21 & 17.65 & 28.92 & 46.57 & 17.16 & 27.05 \\
\hline T5 & $\begin{array}{l}\mathbf{5 0 \%} \mathbf{N}+\operatorname{Reco} . \mathrm{P}+\mathrm{K}+\mathrm{S}+ \\
\mathbf{Z n}+\mathbf{B}+\mathbf{5 0 \%} \mathbf{N} \text { through VC }\end{array}$ & 16.30 & 29.54 & 45.84 & 18.09 & 30.82 & 48.91 & 16.30 & 29.54 \\
\hline T6 & $\begin{array}{l}\mathbf{7 5 \%} \mathbf{N}+\text { Reco. } \mathbf{P}+\mathrm{K}+\mathrm{S}+\mathrm{Zn} \\
+\mathbf{B}+\mathbf{2 5 \%} \mathbf{N} \text { through FYM }\end{array}$ & 15.70 & 24.02 & 39.72 & 16.11 & 26.95 & 43.06 & 15.70 & 24.02 \\
\hline T7 & $\begin{array}{l}75 \% \mathbf{N}+\text { Reco. } P+K+S+Z n \\
+B+25 \% N \text { through } V C\end{array}$ & 17.40 & 26.02 & 43.42 & 17.42 & 27.82 & 45.24 & 17.40 & 26.02 \\
\hline T8 & $\begin{array}{l}50 \% \mathrm{~N}+\text { Reco. } \mathrm{P}+\mathrm{K}+\mathrm{S}+\mathrm{Zn}+ \\
\mathrm{B}+50 \% \mathrm{~N} \text { through FYM } \\
+ \text { Azot }+\mathrm{PSB}\end{array}$ & 17.82 & 30.69 & 49.94 & 18.36 & 32.87 & 51.23 & 17.82 & 30.69 \\
\hline T9 & $\begin{array}{l}50 \% \mathrm{~N}+\text { Reco. } \mathrm{P}+\mathrm{K}+\mathrm{S}+\mathrm{Zn} \\
+\mathrm{B}+50 \% \mathrm{~N} \text { through VC }+ \\
\text { Azot }+ \text { PSB }\end{array}$ & 19.25 & 33.20 & 52.45 & 17.74 & 34.83 & 52.57 & 19.25 & 33.20 \\
\hline T10 & $\begin{array}{l}75 \% \mathrm{~N}+\text { Reco.P }+\mathrm{K}+\mathrm{S}+\mathrm{Zn}+ \\
\mathrm{B}+25 \% \mathrm{~N} \text { through } \mathrm{FYM} \\
+ \text { Azto }+\mathrm{PSB}\end{array}$ & 17.35 & 24.65 & 42.00 & 16.66 & 26.76 & 43.42 & 17.35 & 24.65 \\
\hline T11 & $\begin{array}{l}75 \% \mathrm{~N}+\text { Reco. } \mathrm{P}+\mathrm{K}+\mathrm{S}, \mathrm{Zn}+ \\
\text { B+25\% N through VC }+ \text { Azot } \\
+\mathrm{PSB}\end{array}$ & 17.35 & 24.65 & 42.00 & 16.66 & 26.76 & 43.42 & 17.35 & 24.65 \\
\hline & \pm S.E.(m) & 0.23 & 0.71 & 0.94 & 0.77 & 0.59 & 1.37 & 0.23 & 0.71 \\
\hline & C.D. $(\mathbf{P}=\mathbf{0 . 0 5})$ & 16.82 & 25.91 & 42.73 & 17.51 & 28.07 & 45.58 & 16.82 & 25.91 \\
\hline
\end{tabular}


Table.5 Integrated effect of inorganic, organic and biofertilizers on phosphorus uptake $\left(\mathrm{kg} \mathrm{ha}^{-1}\right)$ in grain, straw and total uptake after harvesting of maize

\begin{tabular}{|c|c|c|c|c|c|c|c|c|c|}
\hline \multirow[t]{2}{*}{ Sr.No } & \multirow[t]{2}{*}{ Treatments } & $\begin{array}{c}\text { Grain } \\
\left(I^{\text {st }}\right. \\
\text { year })\end{array}$ & \multicolumn{2}{|c|}{$\begin{array}{c}\text { Straw } \\
\text { (I It year) }\end{array}$} & $\begin{array}{c}\text { Total } \\
\text { uptake }\end{array}$ & $\begin{array}{r}\text { Grain } \\
\text { (IIId } \\
\text { year) }\end{array}$ & $\begin{array}{l}\text { Strav } \\
\text { (II }{ }^{\mathrm{nc}} \\
\text { year }\end{array}$ & \multicolumn{2}{|c|}{ Total uptake } \\
\hline & & \multicolumn{4}{|c|}{$\left(\right.$ kg ha- $\left.{ }^{1}\right)$} & \multicolumn{4}{|c|}{$\left(\mathrm{kg} \mathrm{ha}^{-1}\right)$} \\
\hline T1 & Control & 5.19 & 12.89 & 18.08 & 5.96 & 13.75 & 19.71 & 5.19 & 12.89 \\
\hline T2 & Recommended NPK & 7.84 & 13.27 & 21.11 & 8.80 & 14.27 & 23.07 & 7.84 & 13.27 \\
\hline T3 & Reco. $\mathrm{NPK}+\mathrm{S}+\mathrm{Zn}+\mathrm{B}$ & 8.12 & 16.90 & 25.02 & 9.48 & 17.42 & 26.90 & 8.12 & 16.90 \\
\hline T4 & $\begin{array}{l}\text { 50\% N + Reco. } \mathrm{P}+\mathrm{K}+\mathrm{S}+ \\
\mathrm{Zn}+\mathrm{B}+\mathbf{5 0 \%} \mathrm{N} \text { through } \\
\mathrm{FYM}\end{array}$ & 10.36 & 15.91 & 26.27 & 11.54 & 16.30 & 27.84 & 10.36 & 15.91 \\
\hline T5 & $\begin{array}{l}\mathbf{5 0 \%} \mathbf{N}+\operatorname{Reco.} \mathbf{P}+\mathbf{K}+\mathbf{S}+ \\
\mathbf{Z n}+\mathbf{B}+\mathbf{5 0 \%} \mathbf{N} \text { through VC }\end{array}$ & 12.23 & 16.09 & 28.32 & 12.67 & 16.10 & 28.77 & 12.23 & 16.09 \\
\hline T6 & $\begin{array}{l}75 \% \mathbf{N}+\operatorname{Reco.} \mathbf{P}+\mathbf{K}+\mathbf{S}+\mathrm{Zn} \\
+\mathbf{B}+25 \% \text { N through FYM }\end{array}$ & 10.57 & 16.49 & 27.06 & 11.56 & 17.19 & 28.75 & 10.57 & 16.49 \\
\hline T7 & $\begin{array}{l}\mathbf{7 5 \%} \mathbf{N}+\text { Reco. } \mathbf{P}+\mathrm{K}+\mathrm{S}+\mathrm{Zn} \\
+\mathrm{B}+25 \% \mathrm{~N} \text { through } \mathrm{VC}\end{array}$ & 10.21 & 17.19 & 27.40 & 11.22 & 18.87 & 30.09 & 10.21 & 17.19 \\
\hline T8 & $\begin{array}{l}50 \% \mathrm{~N}+\mathrm{Reco} . \mathrm{P}+\mathrm{K}+\mathrm{S}+\mathrm{Zn} \\
+\mathrm{B}+50 \% \mathrm{~N} \text { through FYM } \\
+ \text { Azot }+\mathrm{PSB}\end{array}$ & 11.71 & 16.19 & 27.90 & 13.12 & 17.67 & 30.79 & 11.71 & 16.19 \\
\hline T9 & $\begin{array}{l}50 \% \mathrm{~N}+\operatorname{Reco.} \mathrm{P}+\mathrm{K}+\mathrm{S}+\mathrm{Zn} \\
+\mathrm{B}+\mathbf{5 0 \%} \mathrm{N} \text { through VC }+ \\
\text { Azot }+ \text { PSB }\end{array}$ & 12.93 & 17.30 & 30.23 & 12.52 & 18.43 & 30.95 & 12.93 & 17.30 \\
\hline T10 & $\begin{array}{l}75 \% \mathrm{~N}+\text { Reco.P }+\mathrm{K}+\mathrm{S}+\mathrm{Zn}+ \\
\mathrm{B}+25 \% \mathrm{~N} \text { through } \mathrm{FYM} \\
+ \text { Azto }+\mathrm{PSB}\end{array}$ & 12.31 & 15.40 & 27.71 & 12.56 & 16.07 & 28.63 & 12.31 & 15.40 \\
\hline T11 & $\begin{array}{l}75 \% \mathrm{~N}+\operatorname{Reco.} \mathrm{P}+\mathrm{K}+\mathrm{S}, \mathrm{Zn} \\
+\mathrm{B}+25 \% \mathrm{~N} \text { through VC }+ \\
\text { Azot }+\mathrm{PSB}\end{array}$ & 11.59 & 12.89 & 24.48 & 11.82 & 13.75 & 25.57 & 11.59 & 12.89 \\
\hline & \pm S.E.(m) & 0.24 & 1.13 & 1.35 & 0.28 & 0.83 & 1.16 & 0.24 & 1.13 \\
\hline & C.D. $(P=0.05)$ & 0.71 & 0.38 & 1.09 & 0.81 & 0.30 & 1.11 & 0.71 & 0.38 \\
\hline
\end{tabular}


Table.6 Integrated effect of inorganic, organic and biofertilizers on phosphorus uptake ( $\left.\mathrm{kg} \mathrm{ha}^{-1}\right)$ in grain, straw and total uptake after harvesting of wheat

\begin{tabular}{|c|c|c|c|c|c|c|c|c|c|}
\hline \multirow{3}{*}{$\begin{array}{c}\text { Sr.No } \\
\text { T1 }\end{array}$} & \multirow[t]{2}{*}{ Treatments } & $\begin{array}{c}\text { Grain } \\
\left(I^{\text {st }}\right. \\
\text { year })\end{array}$ & \multicolumn{2}{|c|}{$\begin{array}{c}\text { Straw } \\
\left(\mathbf{I}^{\text {st }} \text { year }\right)\end{array}$} & \begin{tabular}{|c|} 
Total \\
uptake
\end{tabular} & $\begin{array}{c}\text { Grain } \\
\text { (IIId } \\
\text { year) }\end{array}$ & $\begin{array}{l}\text { Strav } \\
(\text { (II } \\
\text { year }\end{array}$ & \multicolumn{2}{|c|}{$\begin{array}{l}\text { Total } \\
\text { uptake }\end{array}$} \\
\hline & & \multicolumn{4}{|c|}{$\left(\mathrm{kg} \mathrm{ha}^{1}\right)$} & \multicolumn{4}{|c|}{$\left(\mathrm{kg} \mathrm{ha}^{-1}\right)$} \\
\hline & Control & 8.99 & 12.40 & 21.39 & 8.38 & 13.71 & 22.09 & 8.99 & 12.40 \\
\hline T2 & Recommended NPK & 14.42 & 23.74 & 38.16 & 13.97 & 25.13 & 39.1 & 14.42 & 23.74 \\
\hline T3 & Reco. $\mathrm{NPK}+\mathrm{S}+\mathrm{Zn}+\mathrm{B}$ & 15.84 & 25.82 & 41.66 & 15.57 & 27.19 & 42.76 & 15.84 & 25.82 \\
\hline T4 & $\begin{array}{l}\mathbf{5 0 \%} \mathbf{N}+\operatorname{Reco.} \mathrm{P}+\mathrm{K}+\mathrm{S}+ \\
\mathrm{Zn}+\mathrm{B}+\mathbf{5 0 \%} \mathrm{N} \text { through } \\
\text { FYM }\end{array}$ & 17.16 & 27.05 & 44.21 & 17.65 & 28.92 & 46.57 & 17.16 & 27.05 \\
\hline T5 & $\begin{array}{l}\mathbf{5 0 \%} \mathbf{N}+\operatorname{Reco.} P+\mathrm{K}+\mathrm{S}+ \\
\mathrm{Zn}+\mathrm{B}+\mathbf{5 0 \%} \mathrm{N} \text { through } \\
\mathrm{VC}\end{array}$ & 16.30 & 29.54 & 45.84 & 18.09 & 30.82 & 48.91 & 16.30 & 29.54 \\
\hline T6 & $\begin{array}{l}75 \% \mathrm{~N}+\text { Reco. } \mathrm{P}+\mathrm{K}+\mathrm{S} \\
+\mathrm{Zn}+\mathrm{B}+25 \% \mathrm{~N} \text { through } \\
\text { FYM }\end{array}$ & 15.70 & 24.02 & 39.72 & 16.11 & 26.95 & 43.06 & 15.70 & 24.02 \\
\hline T7 & $\begin{array}{l}75 \% \mathrm{~N}+\text { Reco. } \mathrm{P}+\mathrm{K}+\mathrm{S} \\
+\mathrm{Zn}+\mathrm{B}+25 \% \mathrm{~N} \text { through } \\
\mathrm{VC}\end{array}$ & 17.40 & 26.02 & 43.42 & 17.42 & 27.82 & 45.24 & 17.40 & 26.02 \\
\hline T8 & $\begin{array}{l}\mathbf{5 0 \%} \mathrm{N}+\text { Reco. } \mathrm{P}+\mathrm{K}+\mathrm{S} \\
+\mathrm{Zn}+\mathrm{B}+\mathbf{5 0 \%} \mathrm{N} \text { through } \\
\text { FYM +Azot +PSB }\end{array}$ & 19.25 & 30.69 & 49.94 & 18.36 & 32.87 & 51.23 & 19.25 & 30.69 \\
\hline T9 & $\begin{array}{l}50 \% \mathrm{~N}+\text { Reco. } \mathrm{P}+\mathrm{K}+\mathrm{S} \\
+\mathrm{Zn}+\mathrm{B}+\mathbf{5 0 \%} \mathrm{N} \text { through } \\
\mathrm{VC}+\mathbf{A z o t}+\mathrm{PSB}\end{array}$ & 17.82 & 33.20 & 51.02 & 17.74 & 34.83 & 52.57 & 17.82 & 33.20 \\
\hline T10 & $\begin{array}{l}75 \% \mathrm{~N}+\text { Reco.P }+\mathrm{K}+\mathrm{S}+\mathrm{Zn}+ \\
\text { B+ 25\% N through FYM } \\
+ \text { Azto }+\mathrm{PSB}\end{array}$ & 17.35 & 24.65 & 42.00 & 16.66 & 26.76 & 43.42 & 17.35 & 24.65 \\
\hline T11 & $\begin{array}{l}75 \% \mathrm{~N}+\text { Reco. } \mathrm{P}+\mathrm{K}+\mathrm{S}, \\
\mathrm{Zn}+\mathrm{B}+25 \% \mathrm{~N} \text { through } \\
\mathrm{VC}+\text { Azot }+\mathrm{PSB}\end{array}$ & 17.35 & 24.65 & 42.00 & 16.66 & 26.76 & 43.42 & 17.35 & 24.65 \\
\hline & \pm S.E.(m) & 0.23 & 0.71 & 0.94 & 0.77 & 0.59 & 1.37 & 0.23 & 0.71 \\
\hline & C.D. $(P=0.05)$ & 16.82 & 25.91 & 42.73 & 17.51 & 28.07 & 45.58 & 16.82 & 25.91 \\
\hline
\end{tabular}


Table.7 Integrated effect of inorganic, organic and biofertilizers on potassium uptake (kg ha-1) in grain, straw and total uptake after harvesting of maize

\begin{tabular}{|c|c|c|c|c|c|c|c|c|c|}
\hline \multirow[t]{2}{*}{ Sr.No } & \multirow[t]{2}{*}{ Treatments } & $\begin{array}{c}\text { Grain } \\
\left(I^{\text {st }}\right. \\
\text { year })\end{array}$ & \multicolumn{2}{|c|}{$\begin{array}{c}\text { Straw } \\
\left(I^{\text {st }} \text { year }\right)\end{array}$} & $\begin{array}{c}\text { Total } \\
\text { uptake }\end{array}$ & $\begin{array}{c}\text { Grain } \\
\left(I^{\text {nd }} \text { year }\right)\end{array}$ & \multicolumn{2}{|c|}{$\begin{array}{c}\text { Straw } \\
\text { (II }{ }^{\text {nd }} \text { year) }\end{array}$} & $\begin{array}{l}\text { Total } \\
\text { uptake }\end{array}$ \\
\hline & & \multicolumn{4}{|c|}{$\left(\mathrm{kg} \mathrm{ha}^{1}\right)$} & \multicolumn{4}{|c|}{$\left(\mathrm{kg} \mathrm{ha}^{-1}\right)$} \\
\hline T1 & Control & 4.72 & 87.64 & 92.36 & 4.93 & 90.03 & 94.96 & 4.72 & 87.64 \\
\hline $\mathbf{T 2}$ & Recommended NPK & 6.92 & 95.20 & 102.12 & 7.89 & 93.98 & 101.87 & 6.92 & 95.20 \\
\hline T3 & Reco. $\mathrm{NPK}+\mathrm{S}+\mathrm{Zn}+\mathrm{B}$ & 8.37 & 97.99 & 106.36 & 10.03 & 99.91 & 109.94 & 8.37 & 97.99 \\
\hline T4 & $\begin{array}{l}\mathbf{5 0 \%} \mathbf{N}+\text { Reco. } P+\mathbf{K}+\mathbf{S}+\mathrm{Zn}+ \\
\text { B }+50 \% \mathrm{~N} \text { through FYM }\end{array}$ & 11.55 & 99.03 & 110.58 & 11.97 & 100.87 & 112.84 & 11.55 & 99.03 \\
\hline T5 & $\begin{array}{l}\mathbf{5 0 \%} \mathbf{N}+\mathbf{R e c o .} \mathbf{P}+\mathbf{K}+\mathbf{S}+\mathbf{Z n}+ \\
\mathbf{B}+\mathbf{5 0 \%} \mathbf{N} \text { through VC }\end{array}$ & 12.44 & 103.31 & 115.75 & 12.39 & 106.64 & 119.03 & 12.44 & 103.31 \\
\hline T6 & $\begin{array}{l}\text { 75\% N + Reco. P+ K +S +Zn + } \\
\text { B+25\% N through FYM }\end{array}$ & 12.21 & 100.12 & 112.33 & 12.07 & 10.4 .64 & 116.71 & 12.21 & 100.12 \\
\hline T7 & $\begin{array}{l}75 \% \mathrm{~N}+\text { Reco. } \mathrm{P}+\mathrm{K}+\mathrm{S}+\mathrm{Zn} \\
+\mathrm{B}+25 \% \mathrm{~N} \text { through VC }\end{array}$ & 12.20 & 105.82 & 118.02 & 12.52 & 109.71 & 122.23 & 12.20 & 105.82 \\
\hline T8 & $\begin{array}{l}\mathbf{5 0 \%} \mathrm{N}+\mathrm{Reco.} \mathrm{P}+\mathrm{K}+\mathrm{S}+\mathrm{Zn}+\mathrm{B} \\
+\mathbf{5 0 \%} \mathrm{N} \text { through FYM +Azot } \\
+\mathrm{PSB}\end{array}$ & 13.34 & 108.41 & 121.75 & 12.97 & 110.53 & 123.50 & 13.34 & 108.41 \\
\hline T9 & $\begin{array}{l}50 \% \mathrm{~N}+\text { Reco. } \mathrm{P}+\mathrm{K}+\mathrm{S}+\mathrm{Zn} \\
+\mathrm{B}+\mathbf{5 0 \%} \mathrm{N} \text { through VC }+ \text { Azot } \\
+\mathrm{PSB}\end{array}$ & 14.26 & 114.54 & 128.8 & 14.99 & 115.13 & 130.12 & 14.26 & 114.54 \\
\hline T10 & $\begin{array}{l}75 \% \mathrm{~N}+\mathrm{Reco.P}+\mathrm{K}+\mathrm{S}+\mathrm{Zn}+\mathrm{B}+ \\
25 \% \mathrm{~N} \text { through } \mathrm{FYM}+\text { +Azto } \\
+\mathrm{PSB}\end{array}$ & 12.40 & 107.72 & 120.12 & 12.86 & 108.36 & 121.22 & 12.40 & 107.72 \\
\hline T11 & $\begin{array}{l}75 \% \mathrm{~N}+\text { Reco. } \mathrm{P}+\mathrm{K}+\mathrm{S}, \mathrm{Zn}+ \\
\mathrm{B}+25 \% \mathrm{~N} \text { through } \mathrm{VC}+\text { Azot } \\
+\mathrm{PSB}\end{array}$ & 12.87 & 108.52 & 121.39 & 13.42 & 109.57 & 122.99 & 12.87 & 108.52 \\
\hline & \pm S.E.(m) & 0.32 & 1.78 & 2.09 & 0.49 & 1.81 & 2.30 & 0.32 & 1.78 \\
\hline & C.D. $(P=0.05)$ & 0.95 & 5.26 & 6.21 & 1.02 & 5.38 & 6.40 & 0.95 & 5.26 \\
\hline
\end{tabular}


Table.8 Integrated effect of inorganic, organic and biofertilizers on potassium uptake (kg ha-1) in grain, straw and total uptake after harvesting of wheat

\begin{tabular}{|c|c|c|c|c|c|c|c|c|c|}
\hline \multirow{3}{*}{$\begin{array}{c}\text { Sr. No } \\
\text { T1 }\end{array}$} & \multirow[t]{2}{*}{ Treatments } & \multicolumn{2}{|c|}{$\begin{array}{c}\text { Grain } \\
\left(I^{\text {st }} \text { year }\right)\end{array}$} & $\begin{array}{c}\text { Straw } \\
\left(I^{\text {st }} \text { year }\right)\end{array}$ & $\begin{array}{c}\text { Total } \\
\text { uptake }\end{array}$ & $\begin{array}{c}\text { Grain } \\
\left(\text { II }^{\text {nd }}\right. \\
\text { year })\end{array}$ & \multicolumn{2}{|c|}{$\begin{array}{l}\text { Straw } \\
\text { (II } \\
\text { year) }\end{array}$} & $\begin{array}{c}\text { Total } \\
\text { uptake }\end{array}$ \\
\hline & & \multicolumn{3}{|c|}{$\left(\right.$ kg ha- $\left.^{1}\right)$} & & \multicolumn{4}{|c|}{$\left(\mathrm{kg} \mathrm{ha}^{-1}\right)$} \\
\hline & Control & 20.85 & 53.98 & 74.83 & 23.04 & 58.02 & 81.06 & 20.85 & 53.98 \\
\hline $\mathbf{T 2}$ & Recommended NPK & 39.25 & 88.31 & 127.56 & 42.23 & 90.09 & 132.32 & 39.25 & 88.31 \\
\hline T3 & Reco. $\mathrm{NPK}+\mathrm{S}+\mathrm{Zn}+\mathrm{B}$ & 42.15 & 99.14 & 141.29 & 44.99 & 98.93 & 143.92 & 42.15 & 99.14 \\
\hline T4 & $\begin{array}{l}\mathbf{5 0 \%} \mathrm{N}+\text { Reco. } \mathrm{P}+\mathrm{K}+\mathrm{S}+ \\
\mathrm{Zn}+\mathrm{B}+\mathbf{5 0 \%} \mathrm{N} \text { through } \\
\text { FYM }\end{array}$ & 47.57 & 112.58 & 160.15 & 50.79 & 108.92 & 159.71 & 47.57 & 112.58 \\
\hline T5 & $\begin{array}{l}\mathbf{5 0 \%} \mathbf{N}+\text { Reco. } P+K+S+ \\
\mathbf{Z n}+\mathbf{B}+\mathbf{5 0 \%} \mathbf{N} \text { through VC }\end{array}$ & 49.57 & 119.68 & 169.25 & 52.03 & 118.27 & 170.30 & 49.57 & 119.68 \\
\hline T6 & $\begin{array}{l}75 \% \mathrm{~N}+\text { Reco. } \mathrm{P}+\mathrm{K}+\mathrm{S}+\mathrm{Zn} \\
+\mathrm{B}+25 \% \mathrm{~N} \text { through FYM }\end{array}$ & 41.92 & 104.15 & 146.07 & 47.80 & 104.56 & 152.36 & 41.92 & 104.15 \\
\hline $\mathbf{T 7}$ & $\begin{array}{l}75 \% \mathrm{~N}+\text { Reco. } \mathrm{P}+\mathrm{K}+\mathrm{S}+\mathrm{Zn} \\
+\mathrm{B}+25 \% \mathrm{~N} \text { through } \mathrm{VC}\end{array}$ & 45.6 & 118.6 & 164.2 & 46.86 & 113.12 & 159.98 & 45.6 & 118.6 \\
\hline T8 & $\begin{array}{l}50 \% \mathrm{~N}+\text { Reco. } \mathrm{P}+\mathrm{K}+\mathrm{S}+\mathrm{Zn} \\
+\mathrm{B}+\mathbf{5 0 \%} \mathrm{N} \text { through FYM } \\
+ \text { Azot }+\mathrm{PSB}\end{array}$ & 54.3 & 116.97 & 171.27 & 57.99 & 117.39 & 175.38 & 54.3 & 116.97 \\
\hline T9 & $\begin{array}{l}50 \% \mathrm{~N}+\operatorname{Reco.} \mathrm{P}+\mathrm{K}+\mathrm{S}+\mathrm{Zn} \\
+\mathrm{B}+50 \% \mathrm{~N} \text { through } \mathrm{VC}+ \\
\text { Azot }+ \text { PSB }\end{array}$ & 55.02 & 122.6 & 177.62 & 60.74 & 126.81 & 187.55 & 55.02 & 122.6 \\
\hline T10 & $\begin{array}{l}75 \% \mathrm{~N}+\mathrm{Reco.P}+\mathrm{K}+\mathrm{S}+\mathrm{Zn}+ \\
\mathrm{B}+25 \% \mathrm{~N} \text { through FYM } \\
+ \text { Azto }+ \text { PSB }\end{array}$ & 42.35 & 104.26 & 146.61 & 45.17 & 110.56 & 155.73 & 42.35 & 104.26 \\
\hline T11 & $\begin{array}{l}75 \% \mathrm{~N}+\text { Reco. } \mathrm{P}+\mathrm{K}+\mathrm{S}, \mathrm{Zn} \\
+\mathrm{B}+25 \% \mathrm{~N} \text { through VC }+ \\
\text { Azot }+\mathrm{PSB}\end{array}$ & 43.43 & 110.11 & 153.54 & 48.25 & 108.20 & 156.45 & 43.43 & 110.11 \\
\hline & \pm S.E.(m) & 1.22 & 1.93 & 3.16 & 0.97 & 1.73 & 2.69 & 1.22 & 1.93 \\
\hline & C.D. $(P=0.05)$ & 3.61 & 5.76 & 9.37 & 2.87 & 5.13 & 8.00 & 3.61 & 5.76 \\
\hline
\end{tabular}


Fig.1 Integrated effect of inorganic, organic and biofertilizers on $\mathrm{N}$ uptake $\left(\mathrm{kg} \mathrm{ha}^{-1}\right)$ of maizewheat cropping system during 2015-16 and 2016-17
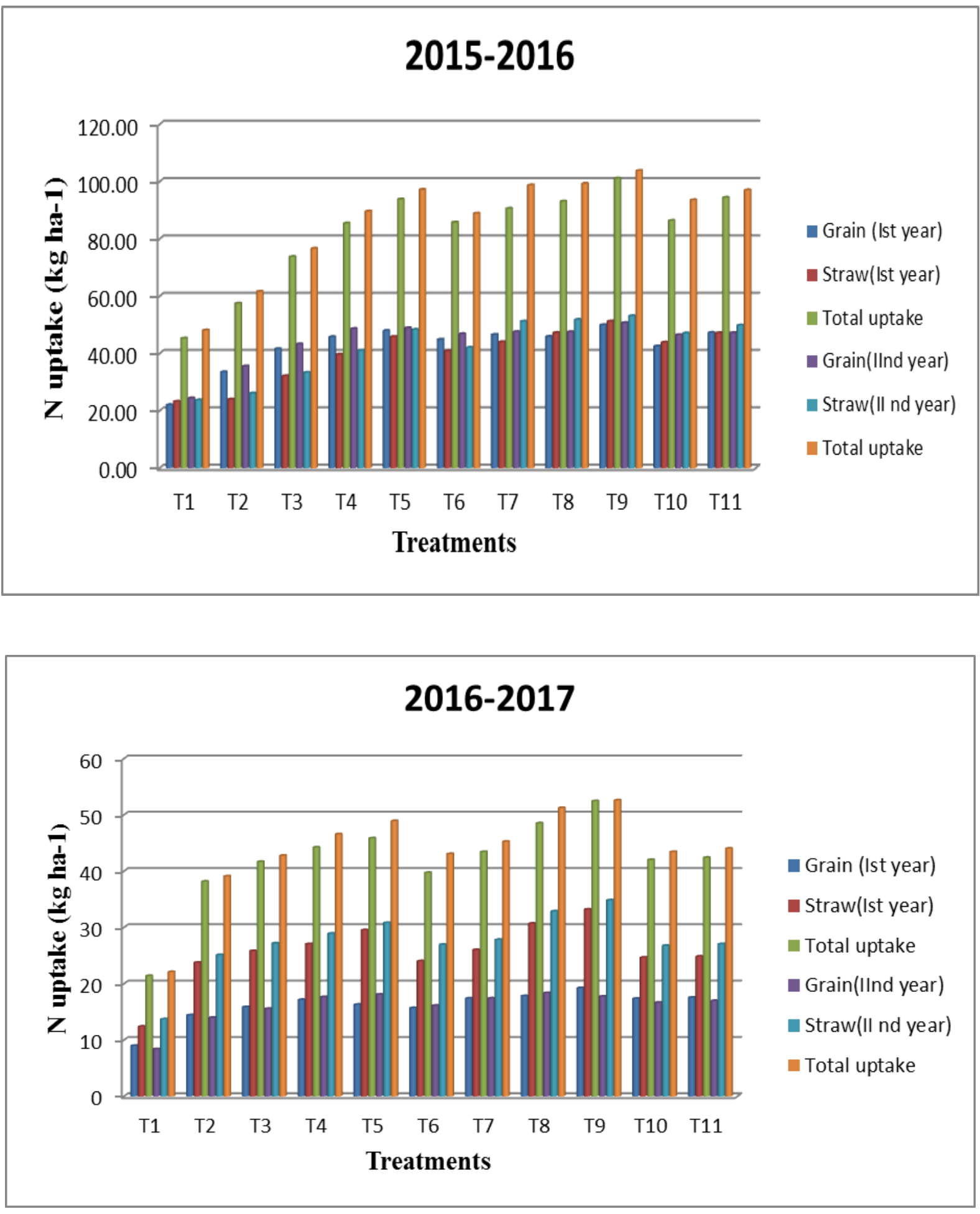
Fig.2 Integrated effect of inorganic, organic and biofertilizers on $\mathrm{P}$ uptake $\left(\mathrm{kg} \mathrm{ha}^{-1}\right)$ of maizewheat cropping system during 2015-16 and 2016-17
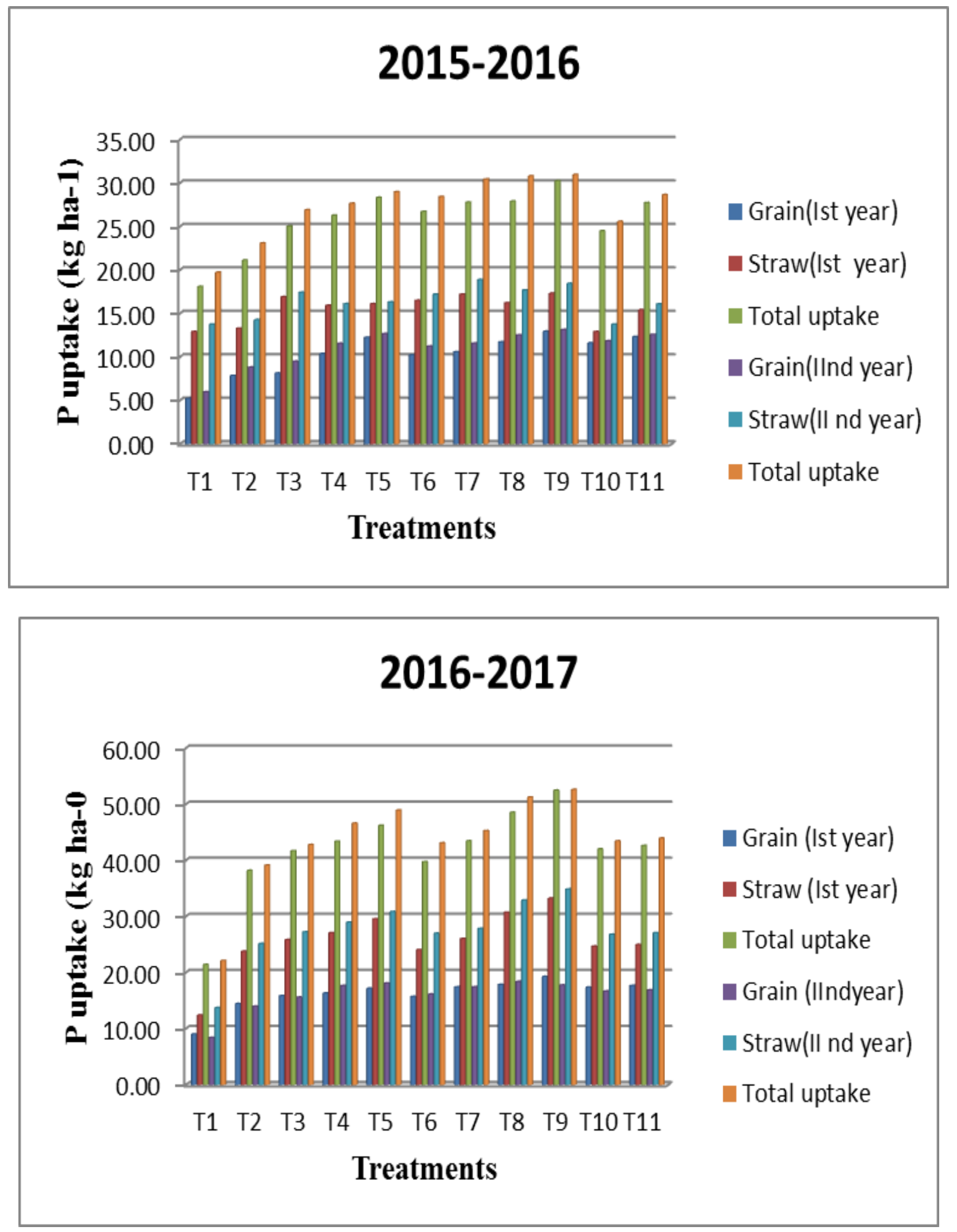
Fig.3 Integrated effect of inorganic, organic and biofertilizers on $\mathrm{K}$ uptake $\left(\mathrm{kg} \mathrm{ha}^{-1}\right)$ of maizewheat cropping system during 2015-16 and 2016-17
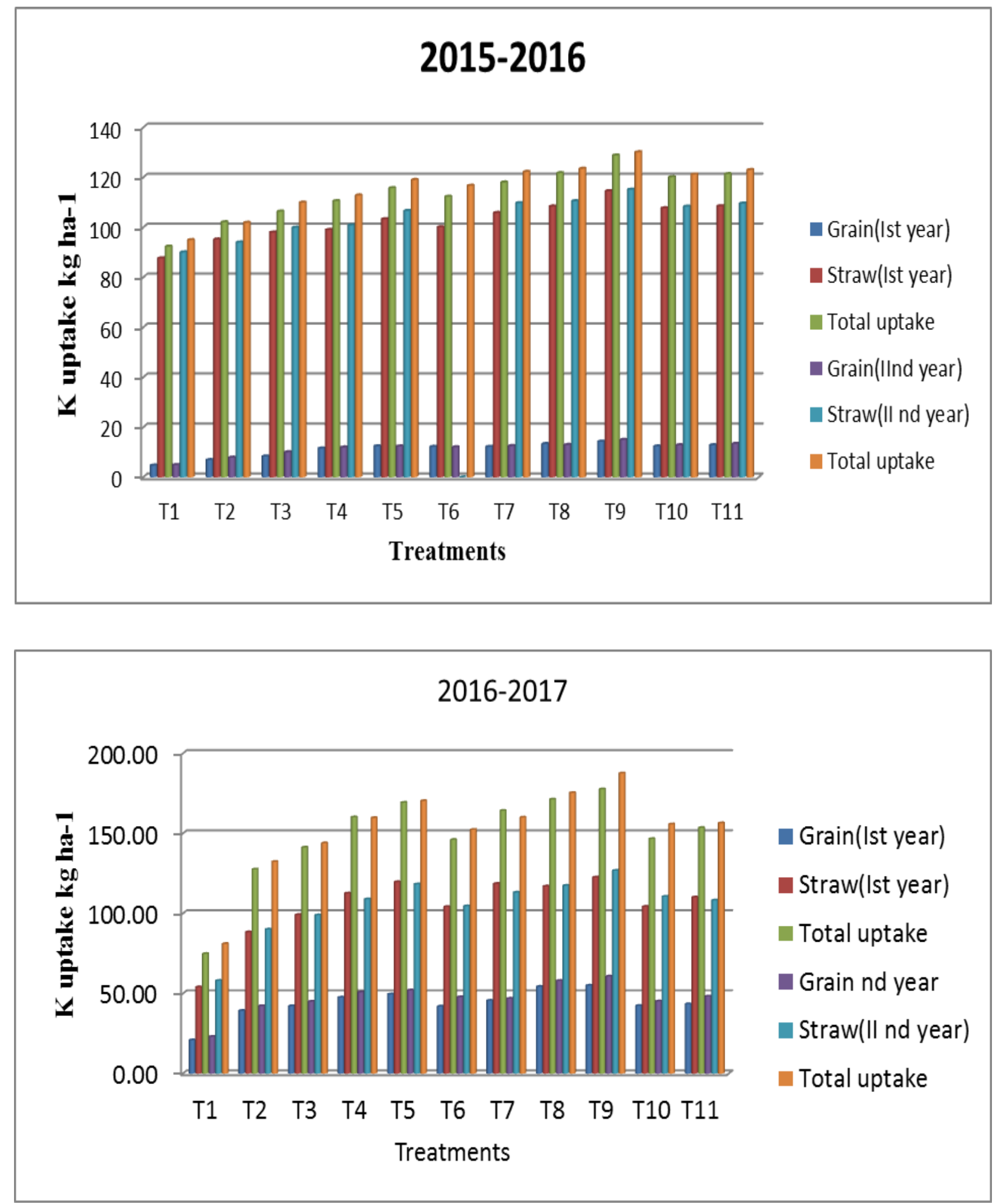
Fig.4 Integrated effect of inorganic, organic and biofertilizers on grain and straw yield ( $q$ ha $\left.^{-1}\right)$ of maize crop during 2015-16
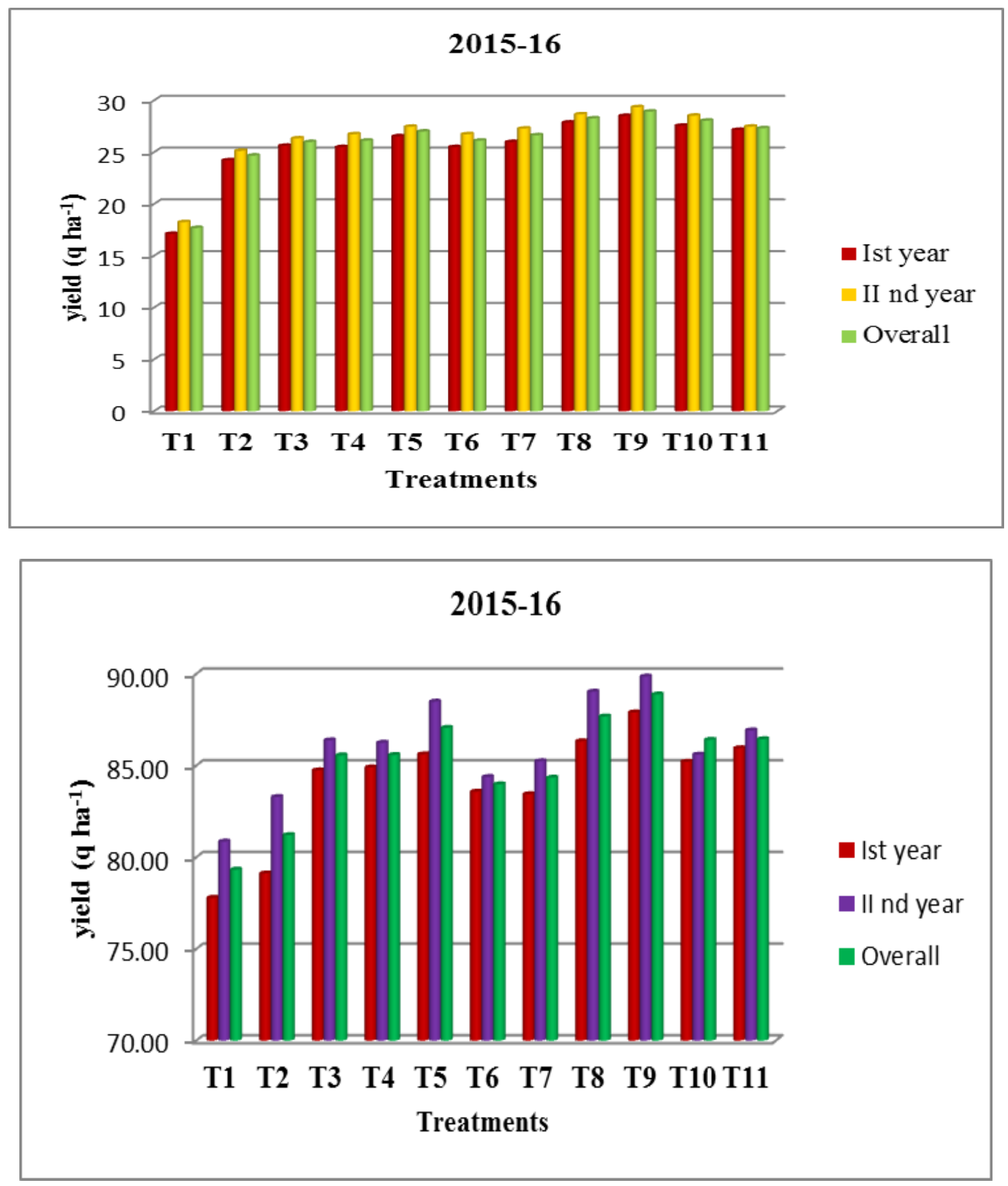
Fig.5 Integrated effect of inorganic, organic and biofertilizers on grain and straw yield (q ha-1) of wheat crop during 2016-17
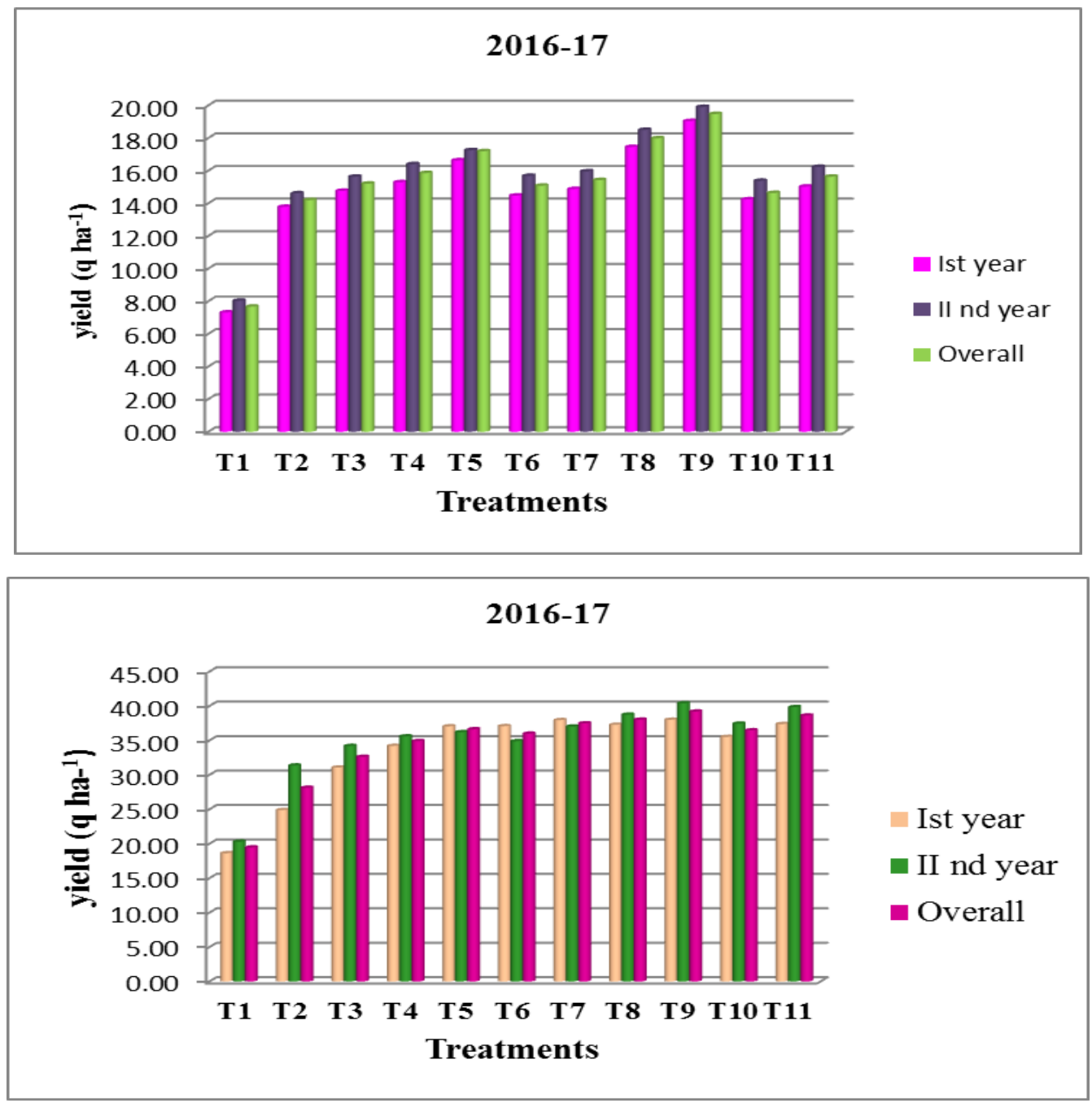

Whereas, lowest $\mathrm{K}$ uptake in $\mathrm{T}_{1}(20.85)$ might be due to scanty availability of $\mathrm{K}$ thereby results decreased its uptake, could be due to balanced application of $\mathrm{N} \mathrm{P} \mathrm{K,} \mathrm{N,} \mathrm{P,} \mathrm{K,} \mathrm{S,} \mathrm{Zn,}$ $\mathrm{B}$ and mixed uses of organic, inorganic and biofertilizers which directly influenced the availability of $\mathrm{K}$ in soils thereby increased $\mathrm{K}$ uptake in all the treatments. These results are line with those reported by Chesti et al., (2013), Khandare et al., (2015) and Thangasamy et al., (2017).

In conclusion, the result of the study concluded with respect to the grain and straw yield of maize influenced significantly due to integration of organic, inorganic and biofertilizers. The values of nutrients uptake viz, N, P, K, S, Zn, B observed highest under $\mathrm{T}_{9}$ consisting integrated uses of organic, inorganic and biofertilizers.

\section{References}

Biswas, S.S., Singhal, S.K., Biswas, D.R., Singh, R.D., Roy, T., Sarkar, A., Ghosh, A. and Das, D. 2017. Synchronization of nitrogen supply with demand by wheat using sewage sludge as organic amendment in an inceptisol. Journal of 
the Indian Society of Soil Science. 65(3): 264-273.

Chesti, M.H., Kohli, A. and Sharma A.K. 2013. Effect of integrated nutrient management on yield of and nutrient uptake by wheat (Triticum aestivum) and soil properties under intermediate zone of Jammu and Kashmir. Journal of the Indian Society of Soil Science. 61(1): $1-6$.

Ghuman, B.S. and Sur, H.S. 2006. Effect of manuring on soil properties and yield of rainfed wheat. Journal of the Indian Society of Soil Science. 54(1): 6-11

Jockson, M.L. (1973) Soil Chemical Analysis, Prentice hall of India Pvt. Limited.

John, M.K., Chuah, H.M. and Neugold, J.H.1975. Application of improved Azomethine-H method is determination of boron in soils and plants. Analytical letters. 8: 550-558

Jadhao, S.D., Arjun, D., Mali, D.V., Singh M., Kharche, V.K., Wanjari, R.H.,Kadu, Sonune, B.A. and Magare, P.N. 2018. Effect of long term manuring and fertilization on depth wise distribution of potassium fractions under sorghumwheat cropping system in vertisol. Journal of the Indian Society of Soil Science. 66(2): 172-181.

Kamble, B.M, Kathmale, D.K. and Rathod, S.D. 2018. Soil nutrient status, uptake, yield and economics of groundnut wheat cropping sequence as influenced by organic sources and fertilizers. Journal of the Indian Society of Soil Science. 66(1): 66-75.

Khandare, R.N., Chandra, R., Pareek, N. and Raverkar, K.P. 2015. Effect of varying rates and methods of carrier based and liquid Azotobacter and PSB biofertilizers on yield and nutrient uptake by wheat (Triticum aestivum L.) and soil properties. Journal of the Indian Society of Soil Science. 63(4): 436-441.
Kour, S., Gupta, M., Kachroo, D., and Bharat, R. 2017. Direct and residual effect of zinc and boron on productivity, nutrient uptake and quality on mustard (Brassica juncea) and succeeding maize (Zea mays) in subtropical Inceptisols of Jammu. Journal of the Indian Society of Soil Science. 65(3): 334-340.

Lindsay, W.L. and Norwell, W.A. 1978. Development of DTPA soil test for zinc, iron, manganese and copper. Soil Science Society of America Journal 42: 421-428.

Olsen, S.R., Cole, C.V., Watanable, F.S. and Dean, L.A. 1994. Extermination of available phosphorus in soils by extraction with sodium bicarbonate. USDA Circular 939.

Mishra, B., Sharma, A., Singh, S.K., Singh, Prasad, J. and Singh, B.P. 2008. Influence of continuous application of amendments to maize-wheat cropping system on dynamics of soil microbial biomass in alfisol of Jharkhand. Journal of the Indian Society of Soil Science. 56 (1):71-75.

Olsen, S.R., Cole, C.V., Watanable, F.S. and Dean, L.A. 1994. Extermination of available phosphorus in soils by extraction with sodium bicarbonate. USDA Circular 939.

Page, A.L.Miller, R.H. and Keeny, D.R. (eds.) 1982. Agronomy Monograph 9, ASA/SSSA Publication, Madison, Wisconsin.

Piper, C.S. (1966). Soil and plant Analysis. Hans Publisher. Bombay, India.

Pathania, K. and Kaleem, M. 2014. Effect of inorganic and organic sources of nutrients on the uptake of maize and its economics. Journal of Soil and Water Conservation 13(2): 151-154.

Sharma, M. P. Sharma, Mondal, A.K., Bhoye, R. C., Samantha A., Rai1, A.P., Arya1, V.M., and Sharma, K.R. 2017. Soil Characterization of Advance Research 
Centre for Rain Fed Agriculture Farm of Sher-e-Kashmir University of Agricultural Sciences and Technology Rakh-Dhinsar, Jammu- (J\&K). International Journal of Plant \& Soil
Science. 20(1): 1-7, 2017

Subbiah, B. V. and Asija, G. L. 1956. A rapid procedure for the determination of available nitrogen in soils. Current Sciences, 25: 259-68.

\section{How to cite this article:}

Bhoye Ranjanabai Chhagan, M.P. Sharma, K.R. Sharma, Abhijit Samanta, Owais Ali Wani, Dileep Kachroo, Manish Kumar, V.K. Razdan, Vikas Sharma, A.K. Mondal and Arya, V.M. 2019. Impact of Organic, Inorganic and Biofertilizers on Crop Yield and N, P and K Uptake under Rainfed Maize-Wheat Cropping System. Int.J.Curr.Microbiol.App.Sci. 8(04): 25462564. doi: https://doi.org/10.20546/ijcmas.2019.804.297 